\title{
Diverse chemotypes disrupt ion homeostasis in the malaria parasite
}

Adele M. Lehane, ${ }^{\star}$ Melanie C. Ridgway, Eileen Baker and Kiaran Kirk**

Research School of Biology, Australian National University, Canberra, ACT 0200, Australia.

\section{Summary}

The antimalarial spiroindolones disrupt Plasmodium falciparum $\mathrm{Na}^{+}$regulation and induce an alkalinization of the parasite cytosol. It has been proposed that they do so by inhibiting PfATP4, a parasite plasma membrane P-type ATPase postulated to export $\mathrm{Na}^{+}$and import $\mathrm{H}^{+}$equivalents. Here, we screened the 400 antiplasmodial compounds of the open access 'Malaria Box' for their effects on parasite ion regulation. Twenty eight compounds affected parasite $\mathrm{Na}^{+}$and pH regulation in a manner consistent with PfATP4 inhibition. Six of these, with chemically diverse structures, were selected for further analysis. All six showed reduced antiplasmodial activity against spiroindolone-resistant parasites carrying mutations in pfatp4. We exposed parasites to incrementally increasing concentrations of two of the six compounds and in both cases obtained resistant parasites with mutations in pfatp4. The finding that diverse chemotypes have an apparently similar mechanism of action indicates that PfATP4 may be a significant Achilles' heel for the parasite.

\section{Introduction}

Nearly half of the world's population live in malariaendemic regions. With the most virulent of the human malaria parasites, Plasmodium falciparum, having acquired resistance to most of the antimalarial drugs that have been deployed, and with increasing reports that resistance to the front-line artemisinin antimalarials may be developing (Wongsrichanalai and Sibley, 2013; Ashley et al., 2014), there is an urgent need to identify new antimalarial agents. In the last five years, millions of compounds have been tested in high throughput 'whole-cell

Accepted 19 August, 2014. For correspondence.

*E-mail adele.lehane@anu.edu.au; Tel. (+61) 261970066.

**E-mail kiaran.kirk@anu.edu.au; Tel. (+61) 261250421. assays' to identify inhibitors of the in vitro growth of (asexual) blood-stage P. falciparum parasites (Plouffe et al., 2008; Gamo et al., 2010; Guiguemde et al., 2010). This approach has uncovered many novel compounds that show potent antiplasmodial activity in vitro. One such compound is the spiroindolone NITD609 (also known as KAE609; Rottmann et al., 2010; Fig. 1), which in a recently completed Phase II trial was shown to clear parasites rapidly in adults with uncomplicated $P$. vivax or $P$. falciparum malaria (White et al., 2014). NITD609 also possesses in vitro activity against the sexual gametocyte, the parasite form transmitted to mosquitoes (van Pelt-Koops et al., 2012).

The mechanism by which spiroindolones kill the parasite has been investigated. Long-term in vitro drug pressure experiments with NITD609 led to the selection of $P$. falciparum parasites with low-level resistance to the drug (Rottmann et al., 2010). The resistant parasites had acquired mutations in PfATP4 (PF3D7_1211900), a P-type ATPase on the parasite plasma membrane (Rottmann et al., 2010). Although PfATP4 was originally annotated as a $\mathrm{Ca}^{2+}$ pump (Krishna et al., 2001) it has been pointed out (Spillman et al., 2013a) that the protein shows significant sequence similarities to the ENA (exitus natrus) $\mathrm{Na}^{+}$-ATPases which efflux $\mathrm{Na}^{+}$from the cells of various lower eukaryotes including protozoa (e.g. lizumi et al., 2006; Lunde et al., 2007).

The intraerythrocytic $P$. falciparum parasite exerts a tight control over its internal $\mathrm{Na}^{+}$levels, maintaining a cytosolic $\mathrm{Na}^{+}$concentration $\left(\left[\mathrm{Na}^{+}\right]_{\mathrm{i}}\right)$ some 10-fold lower than that in the extracellular medium (Lee et al., 1988; Mauritz et al., 2011; Pillai et al., 2013; Spillman et al., 2013a). In parasites functionally isolated from their host cells the addition, at nanomolar concentrations, of growthinhibiting spiroindolones results in an immediate increase in $\left[\mathrm{Na}^{+}\right]_{i}$, consistent with inhibition of a $\mathrm{Na}^{+}$efflux mechanism (Spillman et al., 2013a). A spiroindolone has also been shown to inhibit $\mathrm{Na}^{+}$-dependent ATPase activity (i.e. ATP hydrolysis) in parasitized-erythrocyte membrane preparations (Spillman et al., 2013a), and parasites with spiroindolone-resistance-conferring mutations in PfATP4 have reduced $\mathrm{Na}^{+}$efflux and an increased resting $\left[\mathrm{Na}^{+}\right]_{i}$ (Spillman et al., 2013a). Together, the available data are consistent with the hypotheses that PfATP4 serves as a $\mathrm{Na}^{+}$efflux pump, that it is a target of the spiroindolone 
<smiles>C[C@@H]1Cc2c([nH]c3cc(F)c(F)cc23)[C@@]2(N1)C(=O)Nc1ccc(Cl)cc12</smiles>

NITD246<smiles>C[C@@H]1Cc2c([nH]c3cc(Cl)c(F)cc23)[C@@]2(N1)C(=O)Nc1ccc(Cl)cc12</smiles>

Fig. 1. Chemical structures of the antiplasmodial spiroindolones NITD609 and NITD246. NITD609 is also known as KAE609. antimalarials, and that resistance-conferring mutations in PfATP4 result in reduced sensitivity of the protein to inhibition by the spiroindolones, as well as some impairment of function.

In addition to dissipating the $\mathrm{Na}^{+}$gradient across the plasma membrane of isolated parasites, the antiplasmodial spiroindolones cause an increase in the parasite's cytosolic $\mathrm{pH}\left(\mathrm{pH}_{\mathrm{i}}\right)$, thereby increasing the $\mathrm{pH}$ gradient across the parasite plasma membrane (Spillman et al., 2013a). It has been postulated that PfATP4 expels $\mathrm{Na}^{+}$ from the parasite in exchange for $\mathrm{H}^{+}$(Spillman et al., 2013a), thereby imposing a significant acid load on the parasite (Spillman et al., 2013b). The alkalinization seen in response to inhibition of the $\mathrm{Na}^{+}$extrusion mechanism has been attributed to the lifting of this acid load (Spillman et al., 2013a). $\mathrm{H}^{+}$entering the cell in exchange for $\mathrm{Na}^{+}$, via the putative $\mathrm{Na}^{+}$-ATPase, is extruded by a second plasma membrane pump, a V-type $\mathrm{H}^{+}$-ATPase which is inhibited by concanamycin A (Saliba and Kirk, 1999). Under conditions in which the $\mathrm{Na}^{+}$-ATPase is operational (and therefore imposing an acid load), inhibition of the V-type $\mathrm{H}^{+}$-ATPase by concanamycin $\mathrm{A}$ results in a marked acidification of the parasite cytosol. If the $\mathrm{Na}^{+}$-ATPase is inactivated (either by an inhibitor or by removal of extracellular $\mathrm{Na}^{+}$) then the acidification seen on addition of concanamycin A is reduced (Spillman et al., 2013b).

Together, an increase in $\left[\mathrm{Na}^{+}\right]_{\mathrm{i}}$, an increase in $\mathrm{pH}_{\mathrm{i}}$, and a decrease in the extent of cytosolic acidification seen on inhibition of the V-type $\mathrm{H}^{+}$-ATPase with concanamycin A can be considered a 'signature' of PfATP4 inhibition.

In a bid to further research into novel antiplasmodial compounds identified in the recent large-scale screens, the Medicines for Malaria Venture (MMV) has compiled the open access 'Malaria Box', a collection of 400 structurally diverse antiplasmodial compounds that were selected from the whole-cell drug screen 'hits' and for which the mechanisms of action are unknown (Spangenberg et al., 2013). Here, we sought to determine whether any of the novel chemotypes contained within the Malaria Box disrupt $\mathrm{Na}^{+}$regulation in asexual blood-stage $P$. falciparum parasites.

\section{Results}

\section{Screening the Malaria Box for inhibitors of parasite $\mathrm{Na}^{+}$regulation}

For the initial screen of the Malaria Box, each of the 400 compounds were tested at a concentration of $1 \mu \mathrm{M}$ for an effect on parasite $\left[\mathrm{Na}^{+}\right]_{\text {. }}$. A $1 \mu \mathrm{l}$ aliquot of a $1 \mathrm{mM}$ DMSO stock of each compound was added to a $1 \mathrm{ml}$ suspension of trophozoite-stage $P$. falciparum parasites, isolated by saponin-permeabilization of the erythrocyte membrane and loaded with the $\mathrm{Na}^{+}$-sensitive fluorescent dye SBFI. The suspension was then monitored for any change in the fluorescence ratio. If, on addition of the compound, there was no change in the fluorescence ratio in the ensuing 2 min another compound was added to the same cells. For those compounds for which their addition resulted in an immediate gradual increase in the fluorescence ratio, signifying an increase in $\left[\mathrm{Na}^{+}\right]_{\mathrm{i}}$, the trace was continued without further additions until the fluorescence ratio levelled off at a new value (see Fig. 2A), after which time the screen was continued with a new batch of cells.

Some compounds gave rise to an 'optical effect', detectable as an abrupt change in the fluorescence ratio (see Fig. 2B), and interpreted as being due to an intrinsic fluorescence of the compound or perhaps a chemical interaction between the compound and the fluorescent $\mathrm{Na}^{+}$indicator. In such cases the cells were exchanged for a new batch of cells before testing additional compounds.

For compounds that were without effect on the fluorescence ratio a maximum of nine such compounds were tested on a single batch of cells before the spiroindolone NITD246 (50 nM; Fig. 1) was added as a positive control to ensure that a 'hit' was still detectable in the cells (see Fig. 2C).

From the initial screen, 24 compounds out of the 400 tested were shown to induce an immediate gradual increase in the fluorescence ratio (Table 1). These hits were all re-tested in subsequent $\mathrm{Na}^{+}$experiments, and their effects confirmed.

Fifty five of the 400 compounds gave rise to optical effects in the initial $\mathrm{Na}^{+}$assays. For many of these com- 
A
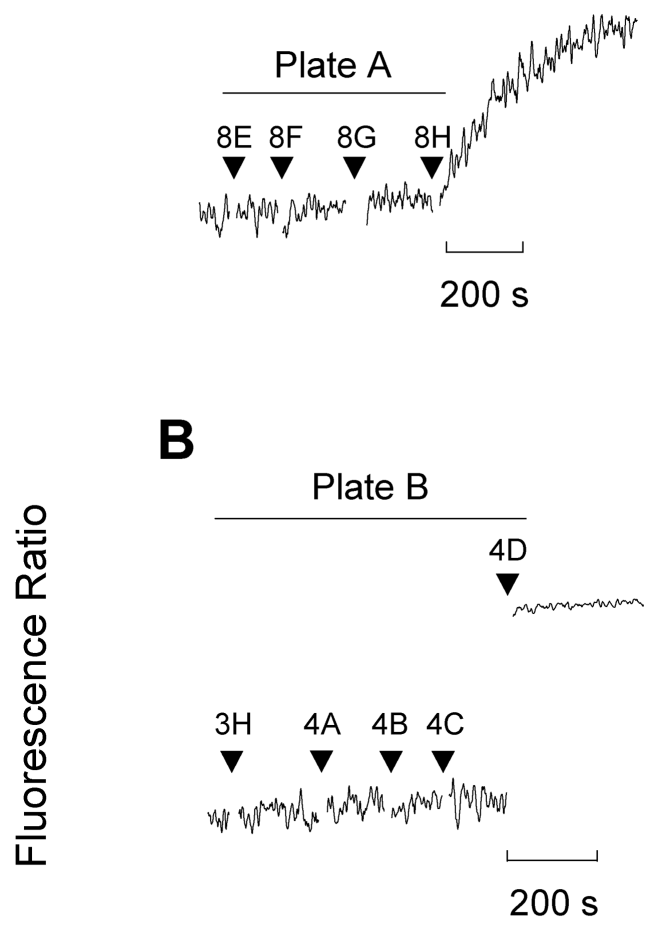

C

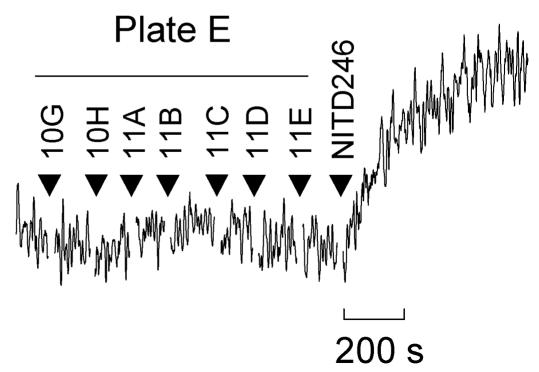

Fig. 2. Representative traces illustrating the strategy used to screen the Malaria Box for inhibitors of parasite $\mathrm{Na}^{+}$regulation. Malaria Box compounds $(1 \mu \mathrm{M})$ were added successively to SBFI-loaded isolated trophozoite-stage parasites until either a hit was detected (A) or a compound caused an optical effect, manifest as an abrupt change in the fluorescence ratio (B). A maximum of nine inactive compounds were tested on a single batch of cells before the spiroindolone NITD246 (50 nM) was added as a positive control to ensure that a hit was still detectable in the cells (C).

pounds it was possible to establish whether or not they were hits in subsequent $\mathrm{Na}^{+}$assays. If an increase in $\left[\mathrm{Na}^{+}\right]_{i}$ by a known hit could be detected in the same cells after the addition of a compound that caused an optical effect, the compound that caused the optical effect was deemed a non-hit. This is because a subsequent hit would not have been detectable had the $\mathrm{Na}^{+}$gradient already been dissipated. Compounds that gave rise to an optical effect and for which a subsequent hit-induced rise in $\left[\mathrm{Na}^{+}\right]_{i}$ could not be detected were tested in $\mathrm{pH}$ experiments (below).

\section{Testing the Malaria Box hits for effects on parasite $\mathrm{pH}_{i}$}

It has been shown previously that compounds such as the spiroindolone NITD246 that inhibit the extrusion of $\mathrm{Na}^{+}$ from the parasite (thereby decreasing the $\mathrm{Na}^{+}$gradient across the parasite plasma membrane) cause an increase in the transmembrane $\mathrm{pH}$ gradient (Spillman et al., 2013a), as well as reducing the extent of acidification that occurs on addition of the $\mathrm{V}$-type $\mathrm{H}^{+}$-ATPase inhibitor concanamycin A (Spillman et al., 2013b). We therefore tested whether the Malaria Box compounds found to cause an increase in $\left[\mathrm{Na}^{+}\right]_{\mathrm{i}}$ in the parasite had an effect on $\mathrm{pH}_{\mathrm{i}}$. Experiments were performed on saponinisolated trophozoite-stage parasites loaded with the $\mathrm{pH}$-sensitive fluorescent dye BCECF.

As expected, the addition of the solvent DMSO at the concentration used to add the test compounds to BCECFloaded parasites suspended in saline solution $(0.1 \% \mathrm{v} / \mathrm{v})$ had no effect on $\mathrm{pH}_{\mathrm{i}}$, and on addition of the $\mathrm{V}$-type $\mathrm{H}^{+}$-ATPase inhibitor concanamycin $\mathrm{A}$ the $\mathrm{pH}_{\mathrm{i}}$ decreased to well below that of the extracellular solution $(\mathrm{pH} \mathrm{7.1;} \mathrm{see}$ representative traces in Fig. 3). As shown previously (Spillman et al., 2013a,b), the addition of NITD246 $(50 \mathrm{nM})$ caused an increase in $\mathrm{pH}_{\mathrm{i}}$ and a reduction in the magnitude of the acidification seen in response to a subsequent addition of concanamycin A (Fig. 3). All 24 of the Malaria Box hits detected in the initial $\mathrm{Na}^{+}$screen, when added at a concentration of $1 \mu \mathrm{M}$, produced an increase in $\mathrm{pH}_{\mathrm{i}}$ and a reduction in the acidification induced by concanamycin A (see examples in Fig. 3). None of them gave rise to optical effects in the $\mathrm{pH}$ assays.

Plate A of the Malaria Box contains the 40 most potent (growth-inhibitory) 'drug-like' compounds and the 40 most potent (growth-inhibitory) 'probe-like' compounds. At a concentration of $1 \mu \mathrm{M}$, all of the hits on Plate $A$ of the Malaria Box gave rise to $\mathrm{pH}_{\mathrm{i}}$ effects that were similar in magnitude to those induced by $50 \mathrm{nM}$ NITD246 [a maximally effective concentration of this compound (Spillman et al., 2013a)]. For most of the compounds on other plates, the effects at $1 \mu \mathrm{M}$ were not as pronounced as those caused by $50 \mathrm{nM}$ NITD246. The finding of submaximal effects at $1 \mu \mathrm{M}$ for these compounds is consistent with the fact that many of the Malaria Box compounds on plates B-E have $50 \%$ inhibitory concentrations $\left(\mathrm{IC}_{50}\right.$ values) for parasite growth $>1 \mu \mathrm{M}$ (http://www. $\mathrm{mmv}$.org/research-development/malaria-box-supportinginformation). As shown in Fig. 3, increasing the concentration of one of the Plate B hits (9G, MMV665796, which has an $\mathrm{IC}_{50}$ for parasite growth of $1.43 \mu \mathrm{M}$; 
Table 1. The Malaria Box compounds designated as hits in parasite $\mathrm{Na}^{+}$and $\mathrm{pH}$ assays, and their effects on parasite ATP levels (after a 30 min incubation with $1 \mu \mathrm{M}$ of the compound; mean \pm SEM derived from 3-4 independent experiments). The 28 compounds identified as hits belong to 17 unique chemotypes. Those (four) chemotypes that include multiple hit compounds are labelled in the Table as Chemotypes I, II, III and IV.

\section{Location in Malaria Box (May 2012 batch)}

Compound identifier

Structure

$[\text { ATP }]_{i}(\%$ of control)

Optical effect?

Plate A: $9 \%$ Hit Rate

$2 B$

MMV006427<smiles>COc1cc(OC)c(N(C)C(=O)c2cc3c(s2)-c2ccccc2S(=O)(=O)O3)cc1Cl</smiles>

$3 E$

MMV000642

(Chemotype I)

$3 \mathrm{H}$

MMV000662

(Chemotype I)

6F

MMV006429

(Chemotype I)

$8 \mathrm{H}$

MMV011567

(Chemotype II)

$10 \mathrm{H}$

MMV665805

(Chemotype II)

$11 \mathrm{C}$

MMV665878

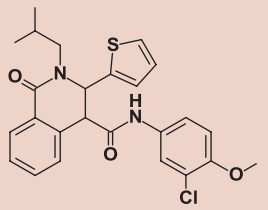

$91 \pm 5$

No<smiles>COc1ccc(NC(=O)C2c3ccccc3C(=O)N(CC(C)C)C2c2cccs2)cc1</smiles>

$91 \pm 0$<smiles>Cc1cc(NC(=O)C2c3ccccc3C(=O)N(CC(C)C)C2c2cccs2)no1</smiles>

$97 \pm 3$<smiles>COc1ccc(C(=O)Nc2nonc2-c2ccc(OC)c(OC)c2)cc1Cl</smiles>

$87 \pm 4$<smiles>CCOc1ccc(-c2n[nH]nc2NC(=O)c2cccc(C)c2)cc1OCC</smiles>

$90 \pm 1$

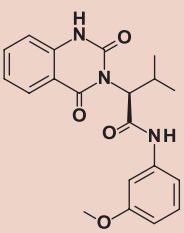

No

No

No

No

No

No 
Table 1. cont.

Location in Malaria Box (May 2012 batch)

Plate B: $11 \%$ Hit Rate

$2 \mathrm{~A}$

$5 G$

$6 \mathrm{~B}$

$8 C$

$8 G$

9F

$9 G$

$10 \mathrm{C}$

$10 \mathrm{H}$
Compound identifier

Structure<smiles>CCOc1cc(Cl)c(C(C)NC(=O)c2ccccc2)cc1OCC</smiles>

$96 \pm 4$

No

(Chemotype III)

MMV000648

(Chemotype I)<smiles>COc1ccccc1CNC(=O)C1c2ccccc2C(=O)N(CC(C)C)C1c1cccs1</smiles>

MMV000653

(Chemotype I)

MMV665918<smiles>CCOc1ccccc1CNC(=O)C1c2ccccc2C(=O)N(CC(C)C)C1c1cccs1</smiles><smiles>O=C(CSc1ccc2nnc(-c3cccc(F)c3)n2n1)Nc1ccc2c(c1)OCO2</smiles>

$98 \pm 4$

No

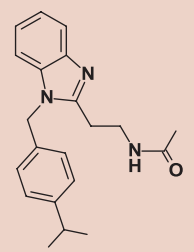

MMV665803

(Chemotype III)

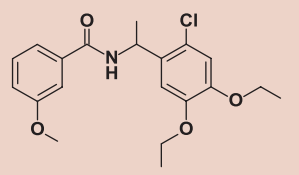

$87 \pm 2$

No

MMV665796

(Chemotype III)

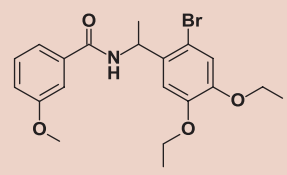

$90 \pm 4$

No

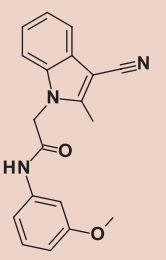

$94 \pm 4$

No

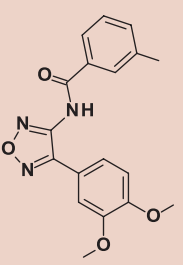

Optical effect?

No

No

No

MMV665826

MMV665890

(Chemotype II)

$[\mathrm{ATP}]_{\mathrm{i}}(\%$ of control)

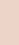


Table 1. cont.

\begin{tabular}{|c|c|c|c|c|}
\hline Location in Malaria Box (May 2012 batch) & Compound identifier & Structure & {$[A T P]_{i}(\%$ of control) } & Optical effect? \\
\hline \multicolumn{5}{|l|}{ Plate C: $5 \%$ Hit Rate } \\
\hline $3 \mathrm{C}$ & $\begin{array}{l}\text { MMV396719 } \\
\text { (Chemotype IV) }\end{array}$ & & $85 \pm 3$ & Yes (SBFI) \\
\hline $3 E$ & $\begin{array}{l}\text { MMV396715 } \\
\text { (Chemotype IV) }\end{array}$ & & $91 \pm 1$ & Yes (SBFI) \\
\hline $5 \mathrm{~F}$ & $\begin{array}{l}\text { MMV396749 } \\
\text { (Chemotype IV) }\end{array}$ & & $93 \pm 5$ & No \\
\hline $10 \mathrm{E}$ & $\begin{array}{l}\text { MMV020660 } \\
\text { (Chemotype II) }\end{array}$ & & $91 \pm 3$ & No \\
\hline \multicolumn{5}{|l|}{ Plate D: $9 \%$ Hit Rate } \\
\hline $2 B$ & MMV665949 & & $96 \pm 4$ & No \\
\hline $2 \mathrm{D}$ & MMV000917 & & $93 \pm 5$ & No \\
\hline $3 \mathrm{H}$ & MMV666025 & & $94 \pm 7$ & No \\
\hline $8 \mathrm{E}$ & MMV008455 & & $95 \pm 4$ & No \\
\hline $8 G$ & MMV006764 & & $94 \pm 3$ & $\begin{array}{l}\text { Yes (SBFI, BCECF } \\
\text { denominator) }\end{array}$ \\
\hline
\end{tabular}


Table 1. cont.

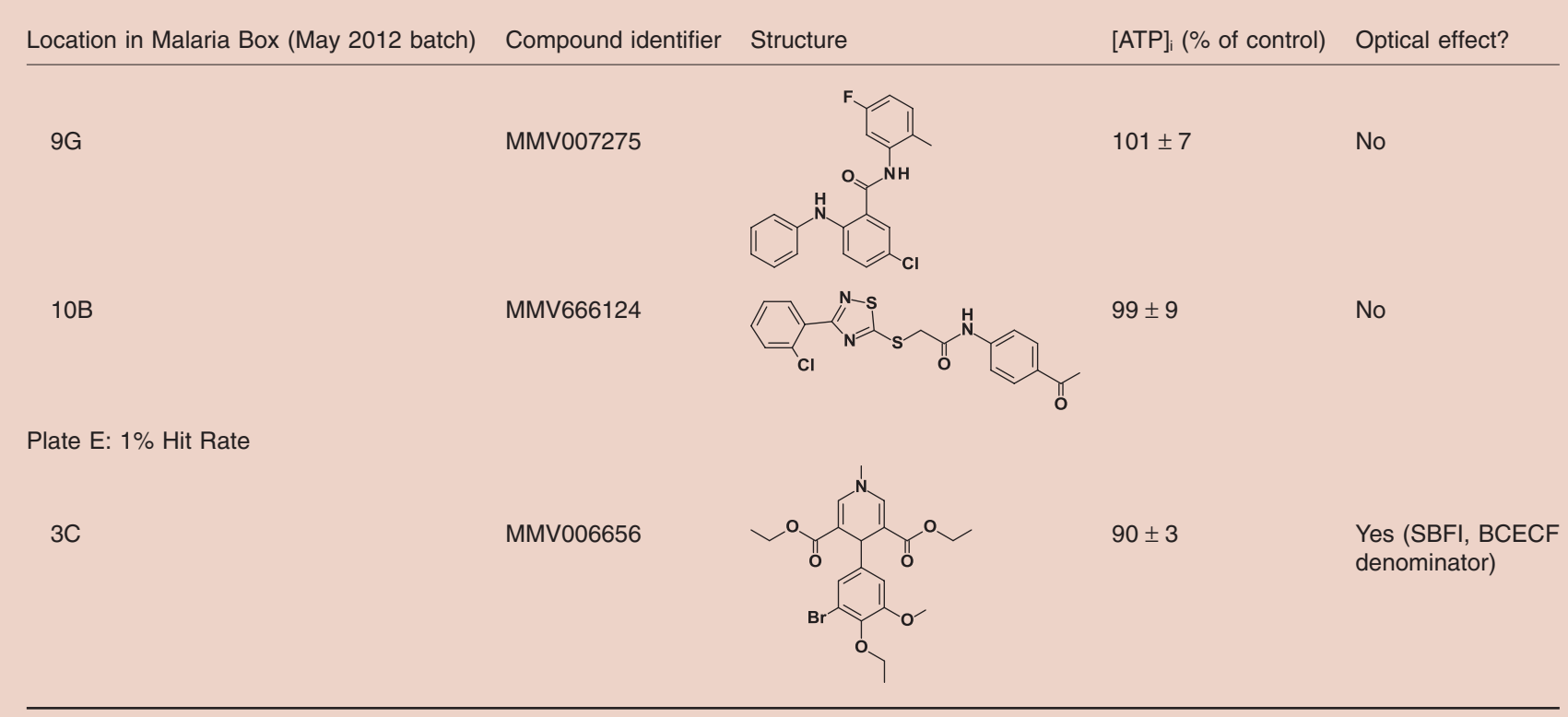

http://www.mmv.org/research-development/malaria-box -supporting-information) from $1 \mu \mathrm{M}$ to $5 \mu \mathrm{M}$ increased its effects on $\mathrm{pH}_{\mathrm{i}}$ towards those seen for 50 nM NITD246.

One of the hits on Plate D (MMV666025) was a weaker hit than all of the others, with even a $5 \mu \mathrm{M}$ concentration giving rise to a submaximal effect in the $\mathrm{pH}$ assay. Furthermore, although compounds similar to MMV666025 are present in the Malaria Box, they were not picked up as hits in our assays. It is possible that if a higher concentration (i.e. $>1 \mu \mathrm{M})$ had been tested in the initial screen $\left(\mathrm{Na}^{+}\right.$ assays), some additional compounds may have caused a detectable increase in fluorescence ratio. However in screening at a concentration of $1 \mu \mathrm{M}$ we were following the recommendation of MMV (http://www.mmv.org/researchdevelopment/malaria-box-faqs), with this recommendation being based on the fact that the vast majority of compounds comprising the Malaria Box have $\mathrm{IC}_{50}$ values for inhibition of parasite growth of $<2 \mu \mathrm{M}$. Thus for any compounds that affected ion regulation only at concentrations higher than $1 \mu \mathrm{M}$ it is unlikely that disruption of ion regulation was their primary mechanism of parasite killing.

We also used the $\mathrm{pH}$ assays to test the compounds that gave rise to optical effects in $\mathrm{Na}^{+}$assays. The majority of these compounds were revealed in the $\mathrm{pH}$ assays to be

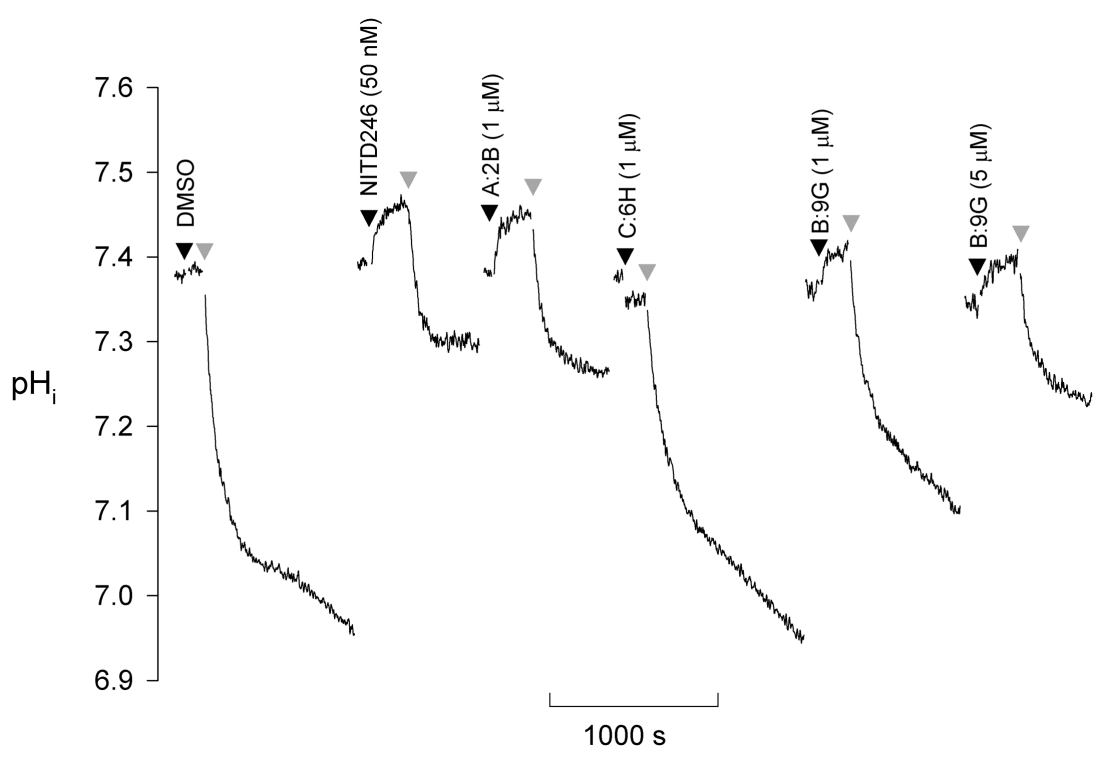

Fig. 3. Representative traces showing the effects of various compounds on $\mathrm{pH}_{\mathrm{i}}$ in isolated trophozoite-stage parasites. DMSO did not increase $\mathrm{pH}_{\mathrm{i}}$, and on addition of concanamycin A (100 nM; grey arrow) $\mathrm{pH}_{\mathrm{i}}$ decreased to below the $\mathrm{pH}$ of the extracellular solution. NITD246 $(50 \mathrm{nM})$ increased $\mathrm{pH}_{\mathrm{i}}$ and reduced the magnitude of the concanamycin A-induced acidification, as did the Plate A hit 2B (MMV006427; $1 \mu \mathrm{M}$ ). A non-hit from Plate $\mathrm{C}(6 \mathrm{H})$ had little effect on $\mathrm{pH}_{\mathrm{i}}$ or concanamycin A-induced acidification. A Plate $B$ hit (9G; MMV665796) had an effect intermediate between that of DMSO and NITD246 at $1 \mu \mathrm{M}$, and a larger effect (similar to that of NITD246) at $5 \mu \mathrm{M}$. 
non-hits, despite some of them giving rise to optical effects in these assays. A compound was considered a non-hit if it had no effect on the $\mathrm{pH}$-sensitive BCECF fluorescence ratio (i.e. the ratio of the fluorescence measured at $520 \mathrm{~nm}$ using the two excitation wavelengths; $490 \mathrm{~nm} / 440 \mathrm{~nm})$, or on the (pH-sensitive) fluorescence measured at $520 \mathrm{~nm}$ following excitation at $490 \mathrm{~nm}$ (i.e. the numerator in the fluorescence ratio), or if it had an abrupt (optical) impact on one or other of these parameters but the subsequent addition of NITD246 or one of the confirmed Malaria Box hits nevertheless caused a detectable increase in the fluorescence ratio (indicative of a cytosolic alkalinization), showing that the compound had not eliminated the acid load associated with $\mathrm{Na}^{+}$ extrusion. There was one compound in the Malaria Box (Plate D, 10D, MMV009127) for which a determination of whether it was a hit or non-hit could not be achieved with either $\mathrm{Na}^{+}$or $\mathrm{pH}$ assays, due to a strong optical effect that resulted in a loss of ion-sensitive fluorescence for both of the fluorescent indicators.

Four compounds that caused optical effects in $\mathrm{Na}^{+}$ experiments were promoted to hit status after the $\mathrm{pH}$ experiments (Table 1). These were $3 \mathrm{C}$ and $3 \mathrm{E}$ on Plate $\mathrm{C}$ (neither gave rise to optical effects in the $\mathrm{pH}$ experiments and both displayed the pre- and post-concanamycin $A$ characteristics of a hit), and $8 G$ on Plate $D$ and $3 C$ on Plate $\mathrm{E}$ (both interfered with the $\mathrm{pH}$-insensitive denominator in the BCECF fluorescence ratio in the $\mathrm{pH}$ experiments, but gave rise to gradual 'hit-like' increases in the $\mathrm{pH}$-sensitive numerator).

In summary, based on the combination of $\mathrm{Na}^{+}$and $\mathrm{pH}$ experiments, 28 out of 400 compounds, equating to $7 \%$ of the Malaria Box compounds, showed evidence of having spiroindolone-like effects on parasite $\mathrm{Na}^{+}$and $\mathrm{pH}$ regulation, consistent with inhibition of PfATP4.

\section{Testing the effects of the Malaria Box hits on parasite ATP levels}

The possibility that the observed disruption of $\mathrm{Na}^{+}$and $\mathrm{pH}$ regulation might be an indirect effect resulting from depletion of parasite ATP was investigated by testing whether any of the hits (at $1 \mu \mathrm{M}$ ) had an effect on parasite ATP levels after a 30 min incubation. Parasites suspended in a glucose-free saline solution were used as a positive control for ATP depletion.

The results, expressed as the intracellular ATP concentration relative to that in control parasites incubated for $30 \mathrm{~min}$ in the presence of the same concentration of DMSO as was used to deliver the inhibitors $(0.1 \% \mathrm{v} / \mathrm{v})$, are shown in Table 1. For all of the hits, the ATP concentration was within $85-101 \%$ of control levels after the $30 \mathrm{~min}$ exposure. Note that $30 \mathrm{~min}$ is much longer than the time required for these compounds to begin disrupting
Table 2. Growth inhibition of the spiroindolone-resistant line NITD609- $R^{\text {Dd2 }}$-clone\#2 and its Dd2 parental line (both from Rottmann et al., 2010) by six Malaria Box compounds, the spiroindolone NITD609, and the antimalarial drugs artemisinin and chloroquine.

\begin{tabular}{lcc} 
& \multicolumn{1}{c}{$\mathrm{IC}_{50}(\mu \mathrm{M})$} \\
\cline { 2 - 3 } Compound & Dd2 parent & NITD609-R ${ }^{\mathrm{Dd} 2}$-clone\#2 \\
\hline MMV011567 & $0.33 \pm 0.03$ & $1.20 \pm 0.20$ \\
MMV665949 & $1.12 \pm 0.13$ & $13.2 \pm 1.9$ \\
MMV007275 & $0.22 \pm 0.02$ & $1.12 \pm 0.10$ \\
MMV006656 & $0.15 \pm 0.01$ & $1.14 \pm 0.07$ \\
MMV006764 & $0.45 \pm 0.05$ & $3.9 \pm 0.4$ \\
MMV665826 & $0.28 \pm 0.04$ & $1.24 \pm 0.15$ \\
NITD609 & $0.00062 \pm 0.00002$ & $0.0111 \pm 0.0002$ \\
Artemisinin & $0.011 \pm 0.001$ & $0.012 \pm 0.002$ \\
Chloroquine & $0.12 \pm 0.02$ & $0.11 \pm 0.02$ \\
\hline
\end{tabular}

The data are from 3 independent experiments and are shown as mean \pm SEM.

$\left[\mathrm{Na}^{+}\right]_{\mathrm{i}}$ and $\mathrm{pH}_{\mathrm{i}}$. There was no significant difference in the intracellular ATP concentration between the control parasites and parasites treated with any of the compounds ( $P>0.05$; one-way ANOVA with post hoc Tukey test).

\section{Testing for cross-resistance between six chemically diverse Malaria Box hits and the spiroindolones}

Six of the Malaria Box hits (those for which additional quantities could be sourced from MMV or from Princeton BioMolecular Research) were tested for antiplasmodial activity against a spiroindolone-resistant line generated by Rottmann et al. (2010) (NITD609-R ${ }^{\text {Dd2 }}$-clone\#2) and its matched Dd2 parental line. The six hits tested (MMV011567, MMV665949, MMV007275, MMV006656, MMV006764 and MMV665826) all belong to distinct chemotypes (Table 1). The spiroindolone-resistant line was significantly less susceptible than the parental line to each of the Malaria Box compounds tested $(P \leq 0.03$; two-tailed paired t-tests; Table 2). The fold difference in the $I_{50}$ values between the two lines ranged from $3.6 \pm 0.3$ (for MMV011567; mean \pm SEM) to $8.9 \pm 1.1$ (MMV006764). There was no difference in the susceptibility of the two lines to either chloroquine or artemisinin $(P \geq 0.1)$.

\section{Generating parasites resistant to the Malaria Box hits MMV011567 and MMV007275}

For each of two chemically distinct Malaria Box hits, MMV011567 and MMV007275, parasite cultures were exposed to increasing concentrations of the compound for a prolonged period (142-150 days) to generate resistant parasites. In the case of MMV011567 results were obtained for two independent duplicate cultures; in the 
Table 3. pfatp4 mutations and growth inhibition by MMV011567, MMV007275 and the spiroindolone NITD609 in parasites generated by prolonged exposure to increasing concentrations of MMV011567 or MMV007275.

\begin{tabular}{lllll} 
& $\begin{array}{l}\text { Mutation in PfATP4 } \\
\text { (relative to Dd2 parent) }\end{array}$ & $\begin{array}{l}\text { MMV011567 } \\
\text { IC } 50 \text { value }(\mu \mathrm{M})\end{array}$ & $\begin{array}{l}\text { MMV007275 } \\
\text { IC50 value }(\mu \mathrm{M})\end{array}$ & NITD609 IC 50 value $(\mu \mathrm{M})$ \\
\hline Dd2 parent & - & $0.34 \pm 0.05$ & $0.23 \pm 0.02$ & $0.00092 \pm 0.00016$ \\
MMV011567-pressured culture 1 & Q172K & $1.63 \pm 0.22$ & nd & $0.0028 \pm 0.0002$ \\
MMV011567-pressured culture 2 & A353Q & $1.89 \pm 0.24$ & nd & $0.0035 \pm 0.0004$ \\
MMV007275-pressured culture & Q172H & nd & $1.10 \pm 0.18$ & $0.0037 \pm 0.0006$ \\
\hline
\end{tabular}

The data are from 3 independent growth inhibition experiments and are shown as mean \pm SEM. nd, no data.

case of MMV007275 results were obtained for a single culture. The experiment was performed with a Dd2 line that had recently been cloned by limiting dilution, and 'drug pressure' (i.e. culturing in the presence of the compound of interest) commenced with the addition of the appropriate compound at its 50\% growth inhibitory concentration $\left(\mathrm{IC}_{50}\right)$ against the $\mathrm{Dd} 2$ clone $(214 \mathrm{nM}$ for MMV011567 and 93 nM for MMV007275). Drug pressure was increased in a stepwise manner: when parasites reached a parasitaemia of $\sim 5 \%$ and the drug $I_{50}$ had increased, the drug concentration was increased to the new $\mathrm{IC}_{50}$.

Table 3 shows $I_{50}$ values for the effects of MMV011567, MMV007275 and NITD609 on the growth of the different parasites, as measured at the end of the drug pressure period. The $\mathrm{IC}_{50}$ values for the effect of MMV011567 on parasites from both of the two MMV011567-pressured cultures were $\sim 5$-fold higher than those of the parental parasites $(P \leq 0.03$; two-tailed paired t-tests). Approximately 5 -fold higher $I_{50}$ values relative to parental parasites were also observed for MMV007275 in parasites from the MMV007275-pressured culture $(P=0.03)$. Parasites from all three of the drug-pressured cultures were also found to be significantly less susceptible to NITD609 than the parental parasites $(P \leq 0.03)$.

Genomic DNA was extracted from each of the drugpressured cultures at the end of the drug pressure period and from the parental Dd2 culture. The entire pfatp4 gene was then sequenced. The parental Dd2 parasites had two polymorphisms in pfatp4 relative to the 3D7 strain (PlasmoDB ID PF3D7_1211900). These were C669A (synonymous) and G3383A (which codes for a G1128R mutation). Parasites from each of the drug-pressured cultures were found to have one additional mutation in pfatp4 (Table 3). Parasites from one of the MMV011567-pressured cultures had a C514A mutation in pfatp4, corresponding to a Q172K mutation in the protein. Parasites from the other MMV011567-pressured culture were found to have a C1058A mutation in pfatp4, corresponding to an A353Q mutation in the protein. Parasites from the MMV007275pressured culture had an A516T mutation in pfatp4, corresponding to a $\mathrm{Q} 172 \mathrm{H}$ mutation in the protein. The three mutations identified here have not been reported previously. The NITD609- $\mathrm{R}^{\mathrm{Dd} 2}$-clone\#2 parasites described by Rottmann et al. (2010) have two mutations in PfATP4: T418N and P990R. I398F, D1247Y, G223R, A184S, I203M and I263V have also been identified in PfATP4 in other spiroindolone-resistant lines, with each line having a maximum of two mutations (Rottmann et al., 2010).

\section{Discussion}

Recent whole-cell assays screening some 4 million compounds from the chemical libraries of St Jude Children's Research Hospital, the Genomics Institute of the Novartis Research Foundation and GlaxoSmithKline have yielded some 20000 inhibitors of the growth in vitro of bloodstage P. falciparum parasites (Plouffe et al., 2008; Gamo et al., 2010; Guiguemde et al., 2010). In compiling the 400-compound Malaria Box, MMV evaluated the ( 5000) commercially available hits with the aim of maximizing the collection's structural diversity (Spangenberg et al., 2013).

The present study revealed that, when added at a concentration of $1 \mu \mathrm{M}, 28$ of the 400 compounds comprising the Malaria Box caused an immediate disruption of malaria parasite ion homeostasis. The nature of the disruption was, in each case, the same as that reported previously for the spiroindolones (Spillman et al., 2013a,b): on addition of the compounds there was, within seconds, an increase in both $\left[\mathrm{Na}^{+}\right]_{\mathrm{i}}$ and $\mathrm{pH}_{\mathrm{i}}$, reflecting a decrease in the $\left[\mathrm{Na}^{+}\right]$gradient and an increase in the $\mathrm{pH}$ gradient across the parasite plasma membrane, with the subsequent inhibition of the parasite's plasma membrane V-type $\mathrm{H}^{+}$-ATPase giving rise to a lesser acidification than was seen under control conditions.

Within these 28 hits there are some compounds with similar structures. Nevertheless the 28 compounds represent 17 unique chemotypes (Table 1). One of these, comprising MMV396719, MMV396715 and MMV396749 on Plate $\mathrm{C}$, is similar in structure to the spiroindolones (Rottmann et al., 2010); however, the compounds comprising the other 16 chemotypes are all quite different in structure from the spiroindolones. 
It has been reported that the spiroindolone NITD609 has a detrimental effect on the sexual gametocyte stage of P. falciparum (van Pelt-Koops et al., 2012). The Malaria Box compounds have recently been screened for antigametocyte activity (Duffy and Avery, 2013; Lucantoni et al., 2013; Bowman et al., 2014). For all but one of the Plate A compounds identified as hits in our study it was reported that a concentration of $5 \mu \mathrm{M}$ reduced the survival of early gametocytes [by $\geq 91 \%$ in the study by Duffy and Avery (2013) and by $\geq 70 \%$ in the study by Lucantoni et al. (2013)]. The one exception, MMV011567, is the least potent of the Plate A hits at killing asexual stage parasites, with an $\mathrm{IC}_{50}$ value $>$ 3.7-fold higher than those for the other compounds (http://www.mmv.org/research-development/ malaria-box-supporting-information). Thus, the lower level of activity against early gametocytes for this compound [a $32 \%$ decrease in survival rate at $5 \mu \mathrm{M}$ in the study by Duffy and Avery (2013) and an 11\% decrease in survival rate at $5 \mu \mathrm{M}$ in the study by Lucantoni et al. (2013)], and for the hits on the other plates (which contain less potent compounds) may reflect a sensitivity issue. The activity of the hits against late-stage gametocytes tended to be lower than their activity against early-stage gametocytes. The average level of growth inhibition at $5 \mu \mathrm{M}$ for our hits was $32 \pm 27 \%$ (mean \pm SD) in the study by Duffy and Avery (2013) and $67 \pm 25 \%$ in the study by Bowman et al. (2014). When considering only the Plate A hits, the level of growth inhibition was $48 \pm 17 \%$ in the study by Duffy and Avery (2013) and $76 \pm 4 \%$ in the study by Bowman et al. (2014).

The observation that all of the hits identified here in the initial $\left[\mathrm{Na}^{+}\right]_{\mathrm{i}}$ assays caused an increase in the transmembrane $\mathrm{pH}$ gradient rules out the possibility that the increase in $\left[\mathrm{Na}^{+}\right]_{i}$ was due to a general disruption of the integrity of the parasite plasma membrane. The finding that none of the hits caused a marked depletion of parasite ATP levels on the timescale of the experiment rules out the possibility of the disruption being a secondary consequence of de-energization of the cell. Instead, the data are consistent with the hypothesis that the compounds identified here bring about a more specific inhibition of parasite $\mathrm{Na}^{+}$extrusion, postulated previously to involve the exchange of $\mathrm{Na}^{+}$for $\mathrm{H}^{+}$equivalents, and to be mediated by PfATP4 (Spillman et al., 2013a,b).

There are a number of alternative mechanisms by which the compounds might inhibit $\mathrm{Na}^{+}$extrusion. One possibility is that the $\mathrm{Na}^{+}$extrusion mechanism is dependent for its activity on a number of other cellular components, and that inhibition of $\mathrm{Na}^{+}$extrusion may be achieved through the interaction of pharmacological agents with any of these. The parasite's putative plasma membrane $\mathrm{Na}^{+}$pump, PfATP4, is closely related to the plasma membrane $\mathrm{Ca}^{2+}$-ATPases (Krishna et al., 2001; Rottmann et al., 2010; Spillman et al., 2013a). Recent work on plasma membrane $\mathrm{Ca}^{2+}$-ATPases has revealed that they are regulated by a diverse range of scaffolding, signalling and regulatory proteins (Strehler, 2013), raising the possibility that the same may be true of PfATP4. Phosphorylation sites have been identified in PfATP4 (Treeck et al., 2011), consistent with kinases being involved in its regulation. Thus, it is possible that the inhibition of $\mathrm{Na}^{+}$extrusion by the diversity of compounds identified here reflects, in at least some cases, an indirect effect.

An alternative possibility is, however, that the inhibition is due in each case to a direct interaction between the compounds identified here and PfATP4. Our finding that each of the six chemically diverse Malaria Box compounds that were identified as hits in the ion regulation assays and then tested in growth assays had reduced antiplasmodial activity against pfatp4 mutant NITD609resistant parasites compared to the matched parental parasites is consistent with this scenario. Furthermore, our finding of pfatp4 mutations in parasites exposed to increasing concentrations of either MMV011567 or MMV007275 is consistent with at least these two compounds targeting PfATP4 itself.

Our study therefore raises the question of why $7 \%$ of a group of compounds selected initially on the basis of their ability to inhibit the growth of $P$. falciparum parasites in in vitro whole-cell assays, and subsequently with a view to providing the broadest cross-section of structural diversity (Spangenberg et al., 2013), might interact with the one protein. For a protein to serve as an effective target in in vitro whole-cell assays such as those used in the initial high throughput screens it is necessary that it be: (i) essential (i.e. necessary for parasite survival), (ii) inhibitable (i.e. able to bind to a pharmacological agent in such a way as to prevent the function of the protein), and (iii) accessible (i.e. able to be accessed by pharmacological agents introduced into the extracellular medium). Perhaps PfATP4 is one of relatively few malaria parasite proteins that, at least in asexual blood-stage parasites grown under in vitro conditions, and perhaps in gametocytes as well, meets these requirements. As such, it may represent a significant Achilles' heel for the parasite.

\section{Experimental procedures}

\section{Compounds}

The Malaria Box was provided by MMV. Each compound was diluted from $10 \mathrm{mM}$ to $1 \mathrm{mM}$ in DMSO and aliquoted into multiple plates. Details of the compounds are available via ChEMBL-NTD (https://www.ebi.ac.uk/chemblntd). The spiroindolone NITD246 (Rottmann et al., 2010), kindly provided by the Novartis Institute for Tropical Diseases (Singapore) through a Material Transfer Agreement, was used as a positive control. NITD609 and additional quantities of the 
Malaria Box compounds MMV011567, MMV007275 and MMV665949 were supplied by MMV. Additional quantities of the Malaria Box compounds MMV006656, MMV006764 and MMV665826 were purchased from Princeton BioMolecular Research.

\section{Parasite culture and isolation}

Unless stated otherwise, all experiments were performed with the chloroquine-resistant Dd2 strain of $P$. falciparum, which is of Thai origin. The spiroindolone-resistant line NITD609- $\mathrm{R}^{\mathrm{Dd} 2}$-clone\#2 generated by Rottmann et al. (2010) and its matched parental line were generously provided by Professor Elizabeth Winzeler. Cultures were maintained as described previously (Trager and Jensen, 1976), with some modifications (Allen and Kirk, 2010), and were synchronized by sorbitol treatment (Lambros and Vanderberg, 1979). Parasites were functionally isolated from their host erythrocytes by exposing the ( $\sim \%$ haematocrit) cultures briefly to saponin $(0.05 \% \mathrm{w} / \mathrm{v}$, of which $\geq 10 \%$ was the active agent sapogenin) (Saliba et al., 1998), and then washing the cells several times in bicarbonate-free RPMI1640 supplemented with $20 \mathrm{mM}$ glucose, $0.2 \mathrm{mM}$ hypoxanthine, $25 \mathrm{mM}$ HEPES, and $25 \mathrm{mg} \mathrm{l}^{-1}$ gentamicin sulphate $(\mathrm{pH} \mathrm{7.10)}$. The isolated parasites were maintained in this medium at $37^{\circ} \mathrm{C}$ and at a density of $1 \times 10^{7}-3 \times 10^{7}$ parasites $\mathrm{ml}^{-1}$ until immediately before their use in either $\mathrm{Na}^{+}$or $\mathrm{pH}$ experiments. For experiments in which parasite ATP levels were measured, the isolated parasites were washed and maintained in 'saline solution' $(125 \mathrm{mM} \mathrm{NaCl}, 5 \mathrm{mM} \mathrm{KCl}, 1 \mathrm{mM} \mathrm{MgCl}$, $20 \mathrm{mM}$ glucose, 25 mM HEPES; pH 7.10) instead of bicarbonate-free RPMI.

\section{Monitoring changes in parasite $\left[\mathrm{Na}^{+}\right]$}

Saponin-isolated trophozoite-stage parasites were loaded with the $\mathrm{Na}^{+}$-sensitive dye SBFI (Molecular Probes, Life Technologies), as described previously (Spillman et al., 2013a). Suspensions of SBFI-loaded, saponin-isolated trophozoites $\left(1 \times 10^{7}-3 \times 10^{7}\right.$ cells $\left.\mathrm{ml}^{-1}\right)$ in saline solution were excited at $340 \mathrm{~nm}$ and $380 \mathrm{~nm}$ successively, and the fluorescence measured at $515 \mathrm{~nm}$, using a PerkinElmer LS 50B Fluorescence Spectrometer with a dual excitation Fast Filter accessory. The ratio of the two measurements $(340 \mathrm{~nm} / 380 \mathrm{~nm})$ was used as an indicator of parasite $\left[\mathrm{Na}^{+}\right]_{\text {. }}$. For the purpose of this study the relationship between fluorescence ratio and $\left[\mathrm{Na}^{+}\right]_{i}$ was not calibrated. The signal-to-noise ratios obtained in the $\mathrm{Na}^{+}$assays varied between experiments, as can be seen from the representative traces shown in Fig. 2.

\section{Measuring parasite $\mathrm{pH}$}

$\mathrm{pH}_{\mathrm{i}}$ was measured at $37^{\circ} \mathrm{C}$ in saponin-isolated trophozoitestage parasites using the ratiometric $\mathrm{pH}$-sensitive fluorescent dye BCECF (Molecular Probes, Life Technologies), as described previously (Saliba and Kirk, 1999). Suspensions of BCECF-loaded, saponin-isolated trophozoites $\left(1 \times 10^{7}-\right.$ $3 \times 10^{7}$ cells $\mathrm{ml}^{-1}$ ) in saline solution were excited at 440 and $490 \mathrm{~nm}$ successively, and the fluorescence measured at $520 \mathrm{~nm}$. The fluorescence measured on excitation at $490 \mathrm{~nm}$ is $\mathrm{pH}$-sensitive whereas that measured on excitation at
$440 \mathrm{~nm}$ is $\mathrm{pH}$-insensitive. The ratio of the two measurements $(490 \mathrm{~nm} / 440 \mathrm{~nm})$ provides an effective measure of cytosolic $\mathrm{pH}$. The relationship between this fluorescence ratio and the cytosolic $\mathrm{pH}$ was calibrated as described previously (Saliba and Kirk, 1999). The signal-to-noise ratios obtained in $\mathrm{pH}$ experiments were typically better than those obtained in $\mathrm{Na}^{+}$experiments ( 10 for the initial alkalinization; cf. Figs 2 and 3).

\section{Measuring parasite ATP levels}

ATP concentrations in isolated trophozoite-stage parasites suspended at $37^{\circ} \mathrm{C}$ at a density of $4 \times 10^{7}-10 \times 10^{7}$ cells $\mathrm{ml}^{-1}$ in saline solution were measured using firefly lantern extract, as described elsewhere (van Schalkwyk et al., 2008).

\section{Parasite proliferation assays}

The effect of compounds on parasite proliferation was assessed using a fluorescent DNA-intercalating dye (Smilkstein et al., 2004), essentially as described previously (Spry et al., 2013). The initial parasitaemia (consisting of predominantly ring-stage parasites) and haematocrit were both $1 \%$, and assays were terminated after $72 \mathrm{~h}$.

\section{Generating and characterizing resistant parasites}

A clone of the chloroquine-resistant Dd2 strain of $P$. falciparum was obtained by limiting dilution, essentially as described previously by Adjalley et al. (2010). For each of two Malaria Box compounds of interest (MMV011567 and MMV007275), flasks containing $1.8 \times 10^{8}$ parasites were exposed to increasing concentrations of the compound for $\sim 5$ months. The starting concentrations of the compounds were their $I_{50}$ values against the clonal Dd2 parasites. For each compound the concentration was increased to the newly determined $\mathrm{IC}_{50}$ value once parasite proliferation was restored and a parasitaemia of $\sim 5 \%$, as well as a higher $I_{50}$ value, were reached. The cultures were provided with fresh medium and blood and diluted to lower the parasitaemia as required.

Genomic DNA was isolated from saponin-isolated parasites by phenol/chloroform extraction with prior Proteinase $\mathrm{K}$ treatment (Gross-Bellard et al., 1973). The entire pfatp4 gene was PCR-amplified using KOD Hot Start DNA polymerase (Novagen) using identical primers to those described previously (Rottmann et al., 2010). The entire pfatp4 gene was sequenced at the Australian Genome Research Facility using the primers used to amplify the gene, the internal sequencing primers described by Rottmann et al. (2010), and three additional internal primers (TATCTCCGTCTTCTACATTATTG, ATGACAGCAATTAATGCAGTTAC and CATGTAGTATATC TGCAACTTTAAC).

\section{Acknowledgements}

We are grateful to Natalie Spillman, Jeremy Burrows and Paul Willis for helpful advice and discussions, to the Canberra Branch of the Australian Red Cross Blood Service for the provision of blood, to Thierry Diagana for the provision of 
NITD246, and to Elizabeth Winzeler for the provision of the NITD609- $R^{D d 2}$-clone\#2 and matched parental Dd2 lines. This work was supported by a grant from MMV and Project Grant 1042272 from the Australian National Health and Medical Research Council (NHMRC). A.M.L. is supported by an NHMRC Overseas Biomedical Fellowship (585519).

\section{References}

Adjalley, S.H., Lee, M.C., and Fidock, D.A. (2010) A method for rapid genetic integration into Plasmodium falciparum utilizing mycobacteriophage Bxb1 integrase. Methods Mol Biol 634: 87-100.

Allen, R.J., and Kirk, K. (2010) Plasmodium falciparum culture: the benefits of shaking. Mol Biochem Parasitol 169: 63-65.

Ashley, E.A., Dhorda, M., Fairhurst, R.M., Amaratunga, C., Lim, P., Suon, S., et al. (2014) Spread of artemisinin resistance in Plasmodium falciparum malaria. N Engl J Med 371: 411-423.

Bowman, J.D., Merino, E.F., Brooks, C.F., Striepen, B., Carlier, P.R., and Cassera, M.B. (2014) Anti-apicoplast and gametocytocidal screening to identify the mechanisms of action of compounds within the Malaria Box. Antimicrob Agents Chemother 58: 811-819.

Duffy, S., and Avery, V.M. (2013) Identification of inhibitors of Plasmodium falciparum gametocyte development. Malar $J$ 12: 408.

Gamo, F.J., Sanz, L.M., Vidal, J., de Cozar, C., Alvarez, E., Lavandera, J.L., et al. (2010) Thousands of chemical starting points for antimalarial lead identification. Nature 465: 305-310.

Gross-Bellard, M., Oudet, P., and Chambon, P. (1973) Isolation of high-molecular-weight DNA from mammalian cells. Eur J Biochem 36: 32-38.

Guiguemde, W.A., Shelat, A.A., Bouck, D., Duffy, S., Crowther, G.J., Davis, P.H., et al. (2010) Chemical genetics of Plasmodium falciparum. Nature 465: 311-315.

lizumi, K., Mikami, Y., Hashimoto, M., Nara, T., Hara, Y., and Aoki, T. (2006) Molecular cloning and characterization of ouabain-insensitive $\mathrm{Na}^{+}$-ATPase in the parasitic protist, Trypanosoma cruzi. Biochim Biophys Acta 1758: 738746.

Krishna, S., Woodrow, C., Webb, R., Penny, J., Takeyasu, K., Kimura, M., and East, J.M. (2001) Expression and functional characterization of a Plasmodium falciparum $\mathrm{Ca}^{2+}$ ATPase (PfATP4) belonging to a subclass unique to apicomplexan organisms. J Biol Chem 276: 10782-10787.

Lambros, C., and Vanderberg, J.P. (1979) Synchronization of Plasmodium falciparum erythrocytic stages in culture. J Parasitol 65: 418-420.

Lee, P., Ye, Z., Van Dyke, K., and Kirk, R.G. (1988) X-ray microanalysis of Plasmodium falciparum and infected red blood cells: effects of qinghaosu and chloroquine on potassium, sodium, and phosphorus composition. Am J Trop Med Hyg 39: 157-165.

Lucantoni, L., Duffy, S., Adjalley, S.H., Fidock, D.A., and Avery, V.M. (2013) Identification of MMV malaria box inhibitors of Plasmodium falciparum early-stage gametocytes using a luciferase-based high-throughput assay. Antimicrob Agents Chemother 57: 6050-6062.
Lunde, C., Drew, D.P., Jacobs, A.K., and Tester, M. (2007) Exclusion of $\mathrm{Na}^{+}$via sodium ATPase (PpENA1) ensures normal growth of Physcomitrella patens under moderate salt stress. Plant Physiol 144: 1786-1796.

Mauritz, J.M., Seear, R., Esposito, A., Kaminski, C.F., Skepper, J.N., Warley, A., et al. (2011) X-ray microanalysis investigation of the changes in $\mathrm{Na}, \mathrm{K}$, and hemoglobin concentration in Plasmodium falciparum-infected red blood cells. Biophys J 100: 1438-1445.

van Pelt-Koops, J.C., Pett, H.E., Graumans, W., van der Vegte-Bolmer, M., van Gemert, G.J., Rottmann, M., et al. (2012) The spiroindolone drug candidate NITD609 potently inhibits gametocytogenesis and blocks Plasmodium falciparum transmission to Anopheles mosquito vector. Antimicrob Agents Chemother 56: 3544-3548.

Pillai, A.D., Addo, R., Sharma, P., Nguitragool, W., Srinivasan, P., and Desai, S.A. (2013) Malaria parasites tolerate a broad range of ionic environments and do not require host cation remodelling. Mol Microbiol 88: 20-34.

Plouffe, D., Brinker, A., McNamara, C., Henson, K., Kato, N., Kuhen, K., et al. (2008) In silico activity profiling reveals the mechanism of action of antimalarials discovered in a highthroughput screen. Proc Natl Acad Sci USA 105: 90599064.

Rottmann, M., McNamara, C., Yeung, B.K., Lee, M.C., Zou, B., Russell, B., et al. (2010) Spiroindolones, a potent compound class for the treatment of malaria. Science 329: 1175-1180.

Saliba, K.J., and Kirk, K. (1999) pH regulation in the intracellular malaria parasite, Plasmodium falciparum: $\mathrm{H}^{+}$ extrusion via a V-type $\mathrm{H}^{+}$-ATPase. $J$ Biol Chem 274: 33213-33219.

Saliba, K.J., Horner, H.A., and Kirk, K. (1998) Transport and metabolism of the essential vitamin pantothenic acid in human erythrocytes infected with the malaria parasite Plasmodium falciparum. J Biol Chem 273: 10190-10195. van Schalkwyk, D.A., Priebe, W., and Saliba, K.J. (2008) The inhibitory effect of 2-halo derivatives of D-glucose on glycolysis and on the proliferation of the human malaria parasite Plasmodium falciparum. J Pharmacol Exp Ther 327: 511-517.

Smilkstein, M., Sriwilaijaroen, N., Kelly, J.X., Wilairat, P., and Riscoe, M. (2004) Simple and inexpensive fluorescencebased technique for high-throughput antimalarial drug screening. Antimicrob Agents Chemother 48: 1803-1806.

Spangenberg, T., Burrows, J.N., Kowalczyk, P., McDonald, S., Wells, T.N., and Willis, P. (2013) The open access malaria box: a drug discovery catalyst for neglected diseases. PLOS ONE 8: e62906.

Spillman, N.J., Allen, R.J., McNamara, C.W., Yeung, B.K., Winzeler, E.A., Diagana, T.T., and Kirk, K. (2013a) $\mathrm{Na}^{+}$ regulation in the malaria parasite Plasmodium falciparum involves the cation ATPase PfATP4 and is a target of the spiroindolone antimalarials. Cell Host Microbe 13: 227237.

Spillman, N.J., Allen, R.J.W., and Kirk, K. (2013b) $\mathrm{Na}^{+}$extrusion imposes an 'acid load' on the intraerythrocytic malaria parasite. Mol Biochem Parasitol 189: 1-4.

Spry, C., Macuamule, C., Lin, Z., Virga, K.G., Lee, R.E., Strauss, E., and Saliba, K.J. (2013) Pantothenamides are potent, on-target inhibitors of Plasmodium falciparum 
growth when serum pantetheinase is inactivated. PLOS ONE 8: e54974.

Strehler, E.E. (2013) Plasma membrane calcium ATPases as novel candidates for therapeutic agent development. J Pharm Pharm Sci 16: 190-206.

Trager, W., and Jensen, J.B. (1976) Human malaria parasites in continuous culture. Science 193: 673-675.

Treeck, M., Sanders, J.L., Elias, J.E., and Boothroyd, J.C. (2011) The phosphoproteomes of Plasmodium falciparum and Toxoplasma gondii reveal unusual adaptations within and beyond the parasites' boundaries. Cell Host Microbe 10: 410-419.

White, N.J., Pukrittayakamee, S., Phyo, A.P., Rueangweerayut, R., Nosten, F., Jittamala, P., et al. (2014) Spiroindolone KAE609 for falciparum and vivax malaria. $N$ Engl J Med 371: 403-410.

Wongsrichanalai, C., and Sibley, C.H. (2013) Fighting drug-resistant Plasmodium falciparum: the challenge of artemisinin resistance. Clin Microbiol Infect 19: 908916. 